\title{
Galunisertib enhances chimeric antigen receptor-modified T cell function
}

\author{
Zhixiong Wang,,${ }^{1,2,3}$ Qian Liu, ${ }^{1}$ Na Risu,,${ }^{3}$ Jiayu Fu, ${ }^{3}$ Yan Zou, ${ }^{4}$ Jiaxing Tang, ${ }^{4}$ Long Li, ${ }^{4}$ Hui Liu,,${ }^{1}$ Guomin Zhou, ${ }^{1}$ \\ Xuekai $\mathbf{Z h u}^{4}$

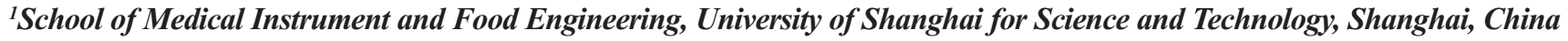 \\ ${ }^{2} M a$ 'anshan University, Ma'anshan, China \\ ${ }^{3}$ Division of Health Science, Graduate School of Medicine, Osaka University, Osaka, Japan \\ ${ }^{4}$ Shanghai Institute for Advanced Immunochemical Studies (SIAIS), ShanghaiTech University, Shanghai, China
}

\begin{abstract}
Chimeric antigen receptor (CAR) T cell therapy still faces the challenge of immunosuppression when treating solid tumors. TGF- $\beta$ is one of the critical factors in the tumor microenvironment to help tumors escape surveillance by the immune system. Here we tried using the combination of a small molecule inhibitor of TGF- $\beta$ receptor I, Galunisertib, and CAR T cells to explore whether Galunisertib could enhance CAR T cell function against solid tumor cells. In vitro experiments showed Galunisertib could significantly enhance the specific cytotoxicity of both CD133- and HER2-specific CAR T cells. However, Galunisertib had no direct killing effect on target cells. Galunisertib significantly increased the cytokine secretion of CAR T cells and T cells that do not express CAR (Nontransfected T cells). Galunisertib did not affect the proliferation of T cells, the antigen expression on target cells and CD69 on CAR T cells. We found that TGF- $\beta$ was secreted by T cells themselves upon activation, and Galunisertib could reduce TGF- $\beta$ signaling in CAR T cells. Our findings can provide the basis for further preclinical and clinical studies of the combination of Galunisertib and CAR T cells in the treatment of solid tumors.
\end{abstract}

Key words: CAR T; TGF- $\beta$; solid tumor; immunotherapy; immunosuppression.

Correspondence: Xuekai Zhu, Shanghai Institute for Advanced Immunochemical Studies (SIAIS), ShanghaiTech University, 393 Middle Huaxia Road, Pudong, Shanghai 201210, China. Tel. +86.021.20685207. E-mail: zhuxk@shanghaitech.edu.cn

Contributions: ZW, QL, XZ conceived the study, designed the experiments, and wrote the paper; HL, GZ, YZ, JT, LL, JF, NR performed experiments and analyzed the data. All authors discussed the results, provided comments, and reviewed the manuscript.

Conflict of interest: The authors declare no competing interests. 


\section{Introduction}

Chimeric antigen receptor (CAR) T cell immunotherapy uses engineered $\mathrm{T}$ cells for recognizing tumor antigens in an HLAindependent manner, thereby killing tumor cells. In 2017, the US Food and Drug Administration (FDA) approved CD19-CAR T cell product Tisagenlecleucel (Novartis) for the treatment of acute lymphoblastic leukemia. ${ }^{1}$ Subsequently, Axicabtagene ciloleucel (Kite Pharma) was approved for the treatment of diffuse large B-cell lymphoma in adults. ${ }^{2}$ In recent years, many attempts of CAR T cell therapy has been made in the treatment of solid tumors such as hepatocellular carcinoma (NCT02715362), ${ }^{3}$ breast cancer, glioma, lung cancer (NCT02713984) ${ }^{4}$ ovarian cancer and pancreatic ductal adenocarcinoma. ${ }^{5}$ However, CAR $\mathrm{T}$ cell therapy still faces many challenges in treating solid tumors. First, it is difficult to obtain ideal tumor-specific targets for CARs. Second, the number of CAR T cells reaching the tumor site is not enough to completely kill the tumor cells after trafficking in vivo. Finally, the tumor microenvironment is generally immunosuppressive, which inhibits CAR T cell function. ${ }^{6}$

TGF- $\beta$ is one of the factors that help tumor cells escape surveillance by the immune system and promote tumor cell proliferation, metastasis, and invasion. ${ }^{7}$ TGF- $\beta$ ligands include TGF- $\beta 1$, TGF- $\beta 2$, and TGF- $\beta 3$. In particular, TGF- $\beta 1$ inhibits IFN $\gamma$ secretion by $\mathrm{CD} 4^{+} \mathrm{T}$ cells ${ }^{8}$ and $\mathrm{CD} 8^{+} \mathrm{T}$ cells, ${ }^{9}$ and proliferation of $\mathrm{CD} 8^{+}$ T cells. ${ }^{10}$

Galunisertib is an oral TGF- $\beta$ receptor type I (TGF- $\beta$ RI) small molecule inhibitor that can regulate $\mathrm{T}$ cell function and improve immunosuppressive tumor microenvironment. Specifically, Galunisertib can reverse the inhibitory effect of TGF- $\beta 1$ on the proliferation of $\mathrm{CD}^{+} \mathrm{T}$ cells and the immunosuppressive effect of Treg cells, and promote the infiltration of $\mathrm{T}$ cells into tumor tissues. ${ }^{10}$ In addition, in 2013, Galunisertib was granted orphan drug designation for the treatment of liver cancer by the European Medical Agency (EMA) and US FDA. Galunisertib is being used in clinical trials for the treatment of hepatocellular carcinoma, glioblastoma, and pancreatic cancer. ${ }^{11}$ Galunisertib may improve the immunosuppression of tumor microenvironment in CAR T cell therapy by regulating $\mathrm{T}$ cell function. At present, researches using the combination of Galunisertib and CAR T cells have not been reported. In this study, we investigated the regulatory effect of Galunisertib on the functions of CD133-CAR T and HER2-CAR T cells when targeting solid tumor cells.

\section{Materials and Methods}

\section{Cell lines}

Human glioma cell line U251, breast cancer cell lines MDAMB-453 and MDA-MB-468 were purchased from the Cell Bank of the Chinese Academy of Sciences (Shanghai, China). The plasmids encoding human CD133 protein and piggyBac transposase were nucleofected into U251 to obtain a glioma cell line with CD133 overexpression (U251 CD133-OE) after drug selection. Similarly, plasmids expressing firefly luciferase and piggyBac transposase were nucleofected into U251 CD133-OE, U251, MDA-MB-453, and MDA-MB-468 cells to acquire U251 CD133OE luc, U251 WT luc, MDA-MB-453 luc, and MDA-MB-468 luc cells. ${ }^{12}$ MDA-MB-453 and MDA-MB-468 were also transduced with lentiviral vectors encoding firefly luciferase, GFP, and puromycin resistance gene (FFluc.-GFP-T2A-Puro, Public Protein/Plasmid Library, Nanjing, China) to obtain cell lines MDA-MB-453 GFP and MDA-MB-468 GFP.

\section{Flow cytometry}

The cells to be stained were collected, centrifuged to remove the medium, and washed once with FACS buffer (PBS $+0.5 \%$ BSA +2 mM EDTA). Then Antibodies were used to label the surface antigens following the instructions provided by the antibody suppliers, and the cells were washed with FACS buffer to remove non-specific binding. Finally, the stained cells were resuspended in FACS buffer and analyzed in a flow cytometer. CAR expression was analyzed with an anti-c-Myc antibody (clone 9B11, 2276S, Cell Signaling Technology, Boston, MA, USA). Antibodies used to detect $\mathrm{T}$ cell differentiation included anti-CD8 (557746, BD Biosciences, Bedford, MA, USA), anti-CD45RO (559865, BD Biosciences), and anti-CD62L (562719, BD Biosciences) antibodies. Tumor antigen expression was measured with antibodies against CD133 (130-098-826, Miltenyi Biotec, Bergisch Gladbach, Germany) or HER2 (BMS120F1, ThermoFisher Scientific, Waltham, MA, USA). Activation markers on CAR T cells were detected by anti-CD69 (555533, BD Biosciences) antibody.

\section{Cytotoxicity assay}

U251 CD133-OE luc (or MDA-MB-453 luc) were co-cultured with CD133-CAR T cells (or HER2-CAR T cells) in plates according to different effector to target ratios. The number of target cells was $1 \times 10^{4}$ per well, and $0.1 \mu \mathrm{M}, 1 \mu \mathrm{M}$, or $10 \mu \mathrm{M}$ Galunisertib was added. As Galunisertib was stocked in DMSO, DMSO was diluted by $10^{3}, 10^{4}$, and $10^{5}$ folds to ensure that the DMSO content in the corresponding control group was equal. And a medium control group (R-10) was also set. After CD133-CAR T cells and U251 CD133-OE luc cells were co-cultured for three days, firefly luciferase substrate was added, and the bioluminescence signal was detected with an Enspire microplate reader (PerkinElmer, Waltham, MA, USA). After $15 \mathrm{~min}$ of continuous measurement, the stable signal value was used to calculate the lysis rate of tumor cells according to the formula: ([signal value of tumor cell alone well - signal value of co-culture well]/signal value of tumor cell alone well). Similarly, after $24 \mathrm{~h}$, the bioluminescence signal in the co-culture of HER2-CAR T cells and MDA-MB-453 luc cells was detected, and the tumor cell lysis rate was calculated too.

To observe the cytotoxicity of HER2-CAR T cells directly, tumor cells expressing GFP (MDA-MB-453 GFP or MDA-MB468 GFP) were seeded into 48-well plate for one day. Then HER2CAR T cells were added according to an effector-to-target ratio of 1:1. 24 hours later, images were taken under microscope (Axio Observer.A1, ZEISS, Oberkochen, Germany).

\section{Real-time cell analysis}

U251 CD133-OE luc or U251 WT tumor cells were seeded in E-plate (ACEA Biosciences, San Diego, CA, USA) at $1 \times 10^{4}$ per well. One day later, CD133-CAR T cells were added to the wells with an effector-to-target ratio of $2: 1$, and $1 \mu \mathrm{M}$ Galunisertib or DMSO was added with the total co-culture volume of $200 \mu \mathrm{L}$. The xCELLigence RTCA instrument (ACEA Biosciences) was used to detect the changes of tumor cells and produce the real-time cellindex curve.

\section{Cytokine secretion}

$2 \times 10^{5}$ CD133-CAR T cells and $1 \times 10^{5} \mathrm{U} 251 \mathrm{CD} 133-\mathrm{OE}$ luc cells were co-cultured in $200 \mu \mathrm{L} \mathrm{R}-10$ in a 96 -well plate; $1 \mu \mathrm{M}$ or $10 \mu \mathrm{M}$ Galunisertib was added to the cells, and DMSO and medium (R-10) were used in control groups. HER2-CAR T cells and their corresponding target cells MDA-MB-453 luc were treated similarly. For NT cells, no tumor cells were added, but Transact was added as activator. In the above three experiments, the supernatant was collected after $24 \mathrm{~h}$, and TNF $\alpha$, GM-CSF, IL-2, and 
IFN $\gamma$ were detected using AlphaLISA kits (TNF $\alpha$, AL208C; GMCSF, AL216C; IL2, AL221C; and IFN $\gamma$, AL217C) from PerkinElmer. TGF- $\beta 1$ secretion was measured by ELISA (EHC107b.96, NeoBioscience, Shanghai, China).

\section{Cell proliferation}

CD133-CAR T cells, HER2-CAR T cells, and NT cells were labeled with $0.5 \mu \mathrm{M}$ CFSE (Carboxyfluorescein Diacetate Succinimidyl Ester, 65-0850-84, ThermoFisher Scientific) according to the method provided by the CFSE manufacturer. Irradiated U251 CD133-OE luc and MDA-MB-453 luc were used as target cells. $1 \times 10^{6} \mathrm{CAR}$ T cells and $5 \times 10^{5}$ target cells were co-cultured in a 24 -well plate $(1 \mathrm{~mL}$ per well). NT cells were activated with Transact. Galunisertib was added, and the controls include DMSO and cell culture medium (R-10). The successful CFSE labeling was confirmed by flow cytometry on the first day, and the CFSE signal was detected again four days later to analyze $\mathrm{T}$ cell proliferation.

\section{Western blot}

$1 \times 10^{6} \mathrm{~T}$ cells were treated with $0.1 \mu \mathrm{M}, 1 \mu \mathrm{M}$, or $10 \mu \mathrm{M}$ Galunisertib for $24 \mathrm{~h}$ in a 48 -well plate, and DMSO was used as control. The cells were collected, washed with PBS, lysed by ultrasound, and boiled in a metal bath. The proteins were separated by SDS-PAGE and transferred onto a PVDF membrane by a semi-dry transfer method. The membrane was blocked with 5\% BSA for $2 \mathrm{~h}$ and incubated with the anti-smad3 (EP823Y, Abcam, Cambridge, UK) or anti-GAPDH (sc-32233, Santa Cruz Biotechnology, Santa Cruz, CA, USA) antibody at $4^{\circ} \mathrm{C}$ overnight. After incubating with Peroxidase-conjugated AffiniPure Donkey Anti-Rabbit IgG (H + L) (711-035-152, Jackson ImmunoResearch Laboratories, West Grove, PA, USA) or Peroxidase-conjugated AffiniPure Donkey Anti-Mouse IgG (H + L) (715-035-150, Jackson ImmunoResearch Laboratories) for $1 \mathrm{~h}$ at room temperature, the proteins were detected with a Bio-Rad ChemiDoc MP chemiluminescence imaging system (Bio-Rad, Hercules, CA, USA).

\section{Statistical analysis}

Statistical analysis of cell lysis rate and cytokine concentration was performed using Prism 6.0 software (GraphPad Software, San Diego, CA, USA). In the significance analysis, one-way ANOVA was used; $* \mathrm{P}<0.05 ; * * \mathrm{P}<0.01 ; * * * \mathrm{P}<0.001 ; * * * * \mathrm{P}<0.0001$.

\section{Results}

\section{Preparation of CD133-CAR T cells and HER2-CAR T cells}

The scFv sequence in CD133-CAR was derived from an antihuman CD133 antibody. The detailed CAR information has been described in our previous work. ${ }^{13}$ Similarly, HER2-CAR piggyBac transposon plasmid was constructed by gene synthesis and conventional molecular cloning methods. Anti-HER2 scFv sequence was from monoclonal antibody clone 4D5. ${ }^{14}$ The piggyBac transposon plasmids were delivered into PBMC by nucleofection. Then T cells were activated with CD3/CD28 magnetic beads (11141D, ThermoFisher Scientific) for the first round of expansion. For the second round of expansion, CAR T cells were activated with CD3 antibody (130-093-387, Miltenyi Biotec, Bergisch Gladbach, Germany), and feeder cells were supplemented. At the end of the expansion, the expression of CAR and the degree of cell differentiation were analyzed by flow cytometry. Taking HER2-CAR T cells as an example, $79.63 \%$ of cells were CD8 positive (Figure 1A), and almost all the cells are CAR positive (Figure 1B) in one representative experiment. Figure $1 \mathrm{C}$ showed the differentiation degree of HER2-CAR T cells, in which central memory $\mathrm{T}$ cells $\left(\mathrm{CD}_{62} \mathrm{~L}^{+} \mathrm{CD} 45 \mathrm{RO}^{+}\right)$and effector memory $\mathrm{T}$ cells $(\mathrm{CD} 62 \mathrm{~L}-$ $\mathrm{CD}^{2} \mathrm{RO}^{+}$) accounted for $42.59 \%$ and $45.65 \%$, respectively.

\section{Galunisertib enhanced the specific cytotoxic ability of CD133- or HER2-CAR T cells}

To investigate the function of Galunisertib on the killing ability of CAR T cells against tumor cells, CD133- or HER2-CAR T cells were co-cultured with the glioma cell line U251 CD133-OE luc or breast cancer cell line MDA-MB-453 luc, respectively. And 0.1 $\mu \mathrm{M}, 1 \mu \mathrm{M}$, or $10 \mu \mathrm{M}$ Galunisertib was added into the co-culture. When the effector to target ratio was 2:1, compared to the DMSO (with the same dilution fold) group, $10 \mu \mathrm{M}$ Galunisertib increased the killing from $50 \%$ to $90 \%$ (Figure $2 \mathrm{~A}$ ). Similarly, compared with the medium control group (R-10), the killing effect was also
A

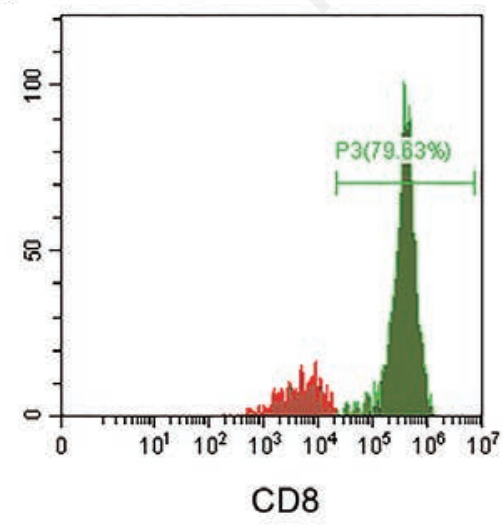

B

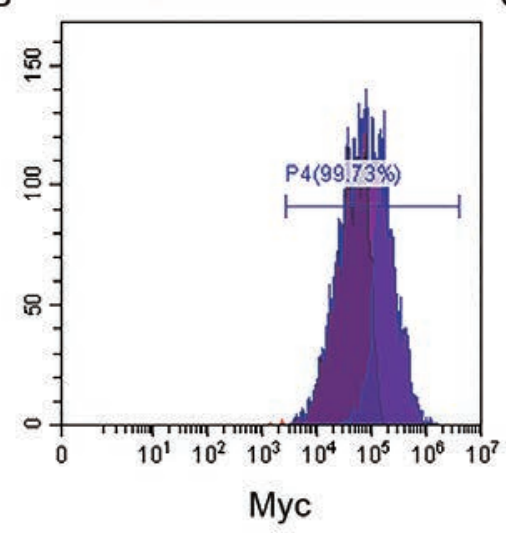

C

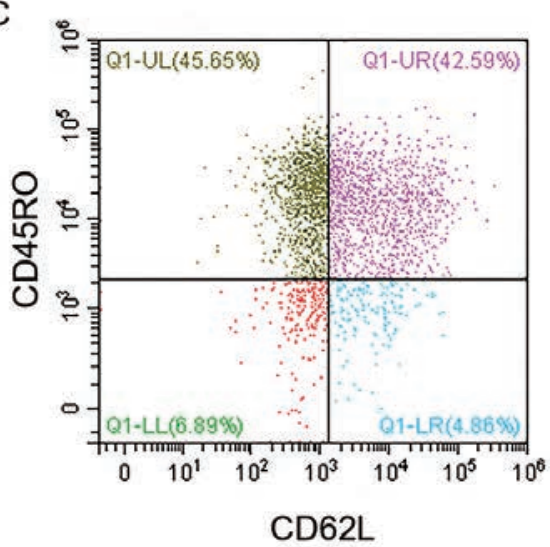

Figure 1. Analysis of the phenotype of HER2-CAR T cells after two rounds of expansion by flow cytometry. HER2-CAR T cells were stained with (A) anti-CD8 antibody, (B) anti-c-Myc antibody, and (C) anti-CD45RO and anti-CD62L antibodies. The negative control was unstained HER2-CAR T cells. 
increased from $70 \%$ to $90 \%$. When the effector to target ratio was $1: 1$, Galunisertib also significantly promoted the killing effect (Figure 2B). DMSO inhibited the cytotoxicity of CD133-CAR T cells slightly (Figure 2 A,B). Furthermore, when the effector to target ratio were $1: 1$ and 1:3, Galunisertib also significantly increased the cytotoxicity of HER2-CAR T cells against MDA-MB-453 luc (Figure $2 \mathrm{D}, \mathrm{E}$ ).

Galunisertib could significantly increase the killing effect of CAR T cells on tumor cells, but it was uncertain whether it is because of Galunisertib regulating CAR T cells or directly killing tumor cells, or inhibiting tumor progression. To answer this question, U251 CD133-OE luc cells and MDA-MB-453 luc cells were treated by Galunisertib or DMSO control alone. Galunisertib had no significant toxicity on either U251 CD133-OE luc cells or MDA-MB-453 luc (Figure 2 C,F). Therefore, Galunisertib enhanced the tumor-killing effect mainly by regulating CAR T cells in the co-culture.

To further study whether the combination of Galunisertib and CAR T cells was also specific for killing tumor cells, tumor cell lines with low expression of antigens were used as target cells. U251 WT luc cells were glioma cells with low expression of
CD133 (Figure 3A). Without Galunisertib in the co-culture, CD133-CAR T cells were able to kill U251 CD133-OE luc cells specifically (Figure 3B). When $1 \mu \mathrm{M}$ Galunisertib was added, cell viability was detected by real-time cell analysis. CD133-CAR T cells could kill U251 CD133-OE luc cells, but had no apparent killing on U251 WT cells (Figure 4A). Similarly, MDA-MB-468 luc cells expressed HER2 at a low level (Figure 3C). Without Galunisertib in the co-culture, HER2-CAR T cells could specifically kill MDA-MB-453 luc cells (Figure 3D). Moreover, to directly view the specific cytotoxicity, tumor cells expressing GFP (MDA-MB-453 GFP or MDA-MB-468 GFP) were used as target cells, and the cultured cells were observed under microscope. As shown in Figure 3E, compared to MDA-MB-453 GFP alone, HER2-CAR T cells and MDA-MB-453 GFP formed clumps, and there were less GFP positive tumor cells in the co-culture well. However, no apparent difference was found between MDA-MB468 GFP alone and the co-culture of HER2-CAR T cells and MDA-MB-468 GFP. When Galunisertib was added, the combination of Galunisertib and HER2-CAR T cells still could only kill MDA-MB-453 luc cells, not MDA-MB-468 luc cells (Figure 4B).
A

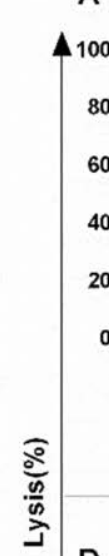

D

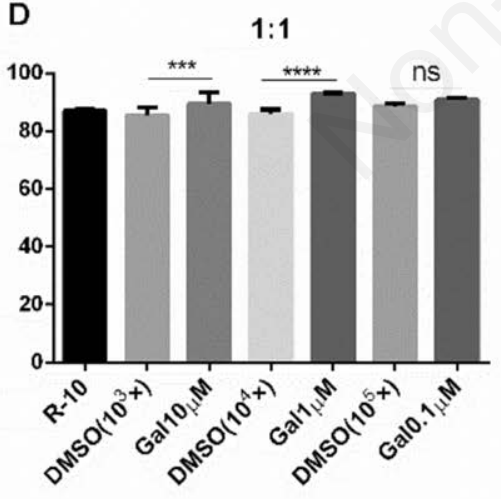

B

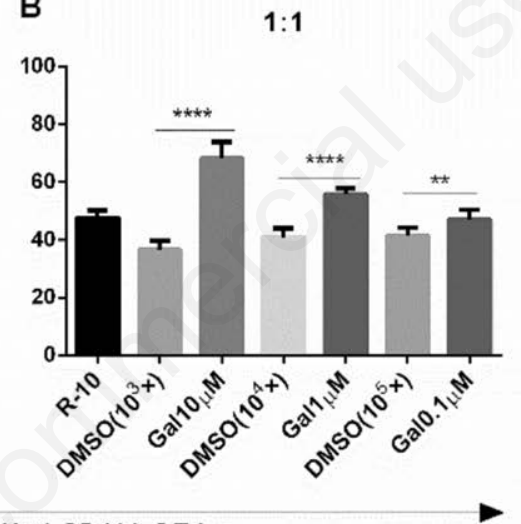

E

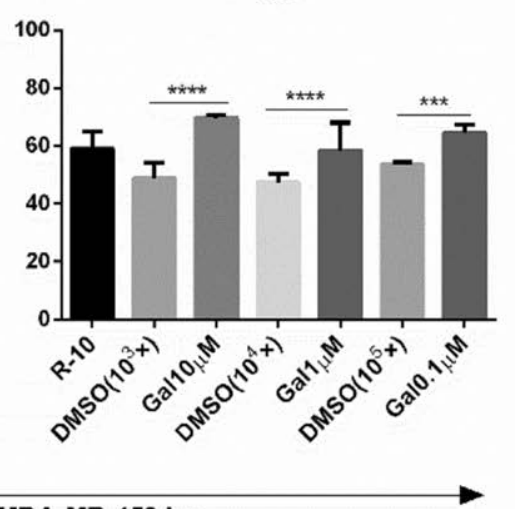

C

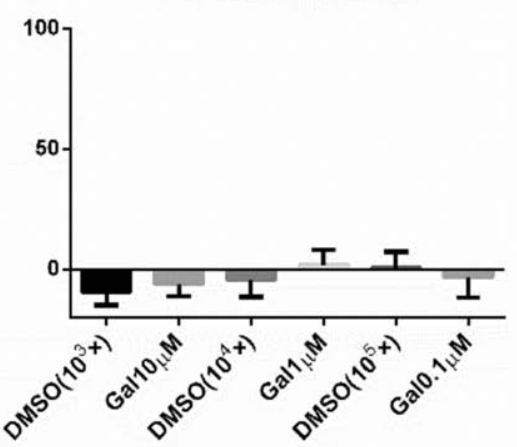

F

MDA-MB-453 luc

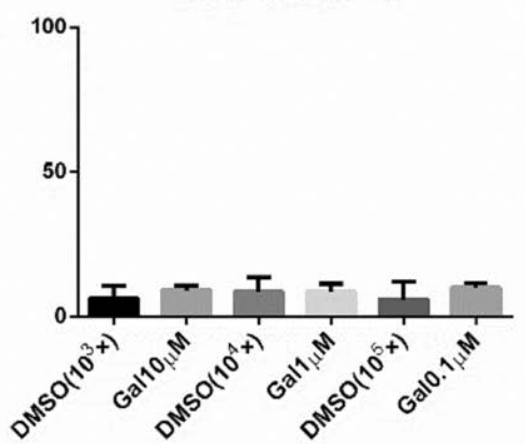

HER2-CAR T+MDA-MB-453 luc

Figure 2. Galunisertib significantly increased the killing of tumor cells by CAR T cells. U251 CD133-OE luc and CD133-CAR T cells were co-cultured in a 96-well plate with an effector to target ratio of (A) 2:1 or (B) 1:1, and Galunisertib was added at the same time, and the cell lysis rate was measured three days later. C) U251 CD133-OE luc cells were treated with Galunisertib alone for three days, and the cell lysis rate was analyzed. MDA-MB-453 luc cells were co-cultured with HER2-CAR T cells with an effector to target ratio of (D) 1:1 or (E) 1:3. Galunisertib was added simultaneously, and the cell lysis rate was analyzed one day later. F) MDA-MB-453 luc was treated with Galunisertib alone, and the cell lysis rate was determined one day later. Gal, $10 \mu \mathrm{M}, 1 \mu \mathrm{M}$, or $0.1 \mu \mathrm{M}$ Galunisertib was added to the culture; DMSO, a DMSO control at the same dilution fold as Galunisertib; R-10, a medium control. 
Galunisertib increased the cytokine secretion of CD133CAR T cells, HER2-CAR T cells, and NT cell

To investigate the effect of Galunisertib on the cytokine secretion of CAR T cells, CD133-CAR T cells were co-cultured with U251 CD133-OE luc. Galunisertib $(1 \mu \mathrm{M}$ or $10 \mu \mathrm{M})$ was added to the coculture, and the control group included DMSO and culture medium R10. Supernatants were collected $24 \mathrm{~h}$ later, and the secretion of TNF $\alpha$, GM-CSF, IL-2, and IFN $\gamma$ was detected by AlphaLISA. The secretion of all four cytokines by CD133-CAR T cells was increased signifi- cantly when Galunisertib concentration was $1 \mu \mathrm{M}$ or $10 \mu \mathrm{M}$ (Figure 5A). A similar method was used to analyze the cytokine secretion of HER2-CAR T cells when co-cultured with MDA-MB-453 luc cells. As shown in Figure 5B, $1 \mu \mathrm{M}$ Galunisertib could significantly enhance the secretion of GM-CSF, IL-2 and IFN $\gamma$, but not TNF $\alpha$. To further study whether Galunisertib's stimulatory effects on cytokine secretion depend on CAR signals, Transact was used to activate NT cells, which did not express CAR. After co-culture with different concentrations of Galunisertib for $24 \mathrm{~h}$, the supernatant was taken, and the
A

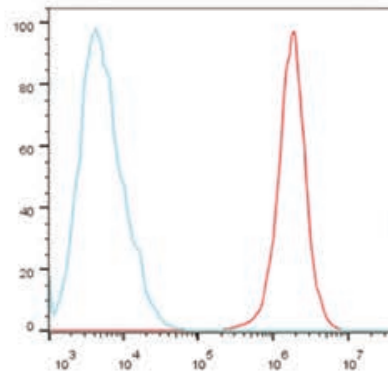

CD133

C
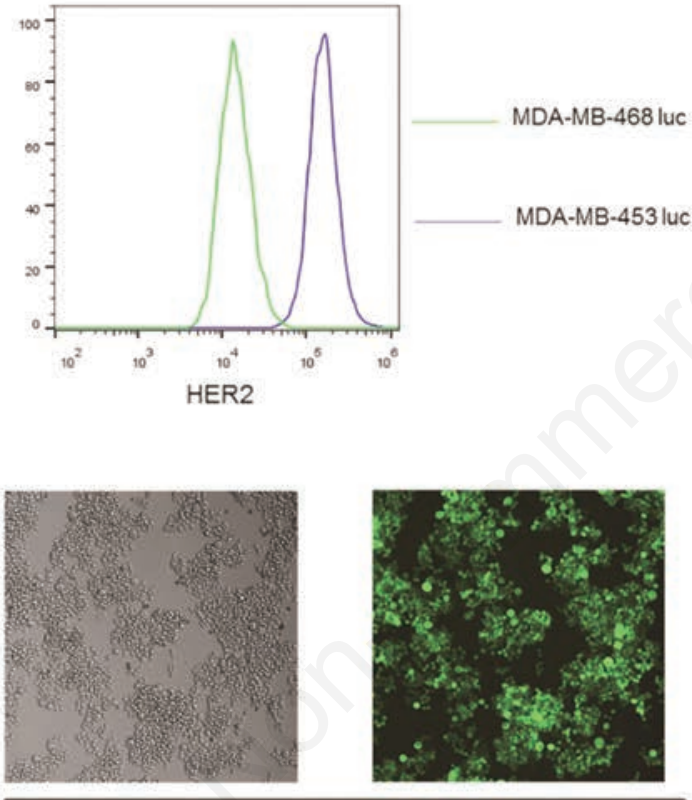

MDA-MB-453 GFP alone
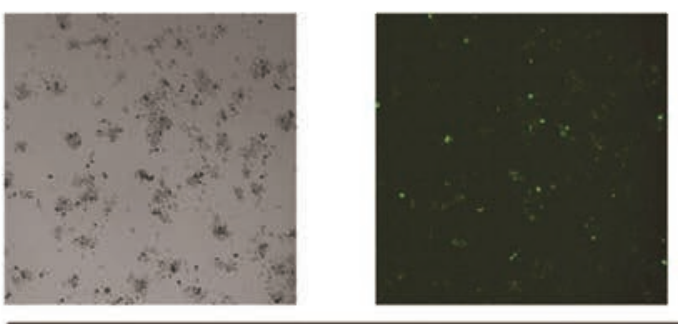

MDA-MA-453 GFP+HER2-CAR T
B

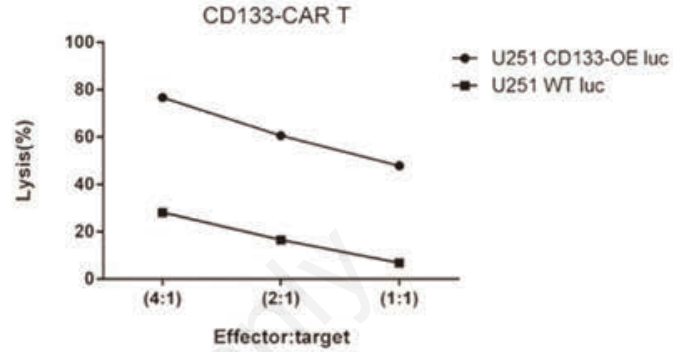

D
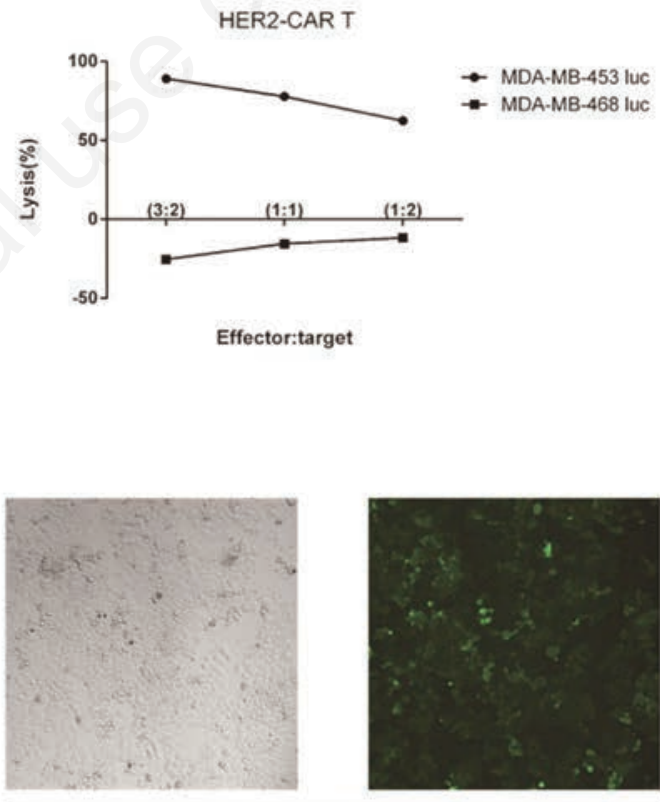

MDA-MB-468 GFP alone

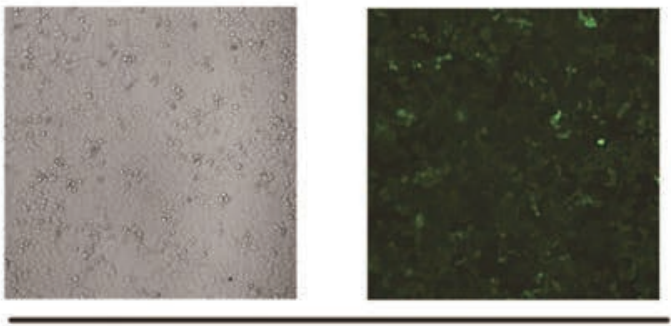

MDA-MB-468 GFP+HER2-CAR T

Figure 3. CAR T cells could specifically kill target cells. A) Flow cytometric analysis of CD133 expression on the surface of target cells. B) According to different effector to target ratios, CD133-CAR T cells were co-cultured with U251 CD133-OE luc or U251 WT luc for three days, respectively, and then tested for lysis rate. C) Flow cytometry analysis of HER2 expression on the surface of target cells. D) HER2-CAR T cells were co-cultured with MDA-MB-453 luc or MDA-MB-468 luc for one day and tested for lysis rate. E) Tumor cells expressing GFP (MDA-MB-453 GFP or MDA-MB-468 GFP) were cultured alone or co-cultured with HER2-CAR T cells for one day. Then images of the cells were taken under microscope. 
cytokines were measured. $1 \mu \mathrm{M}$ Galunisertib could significantly promote the secretion of TNF $\alpha$, GM-CSF, IL-2, and IFN $\gamma$ by NT cells (Figure 5C), which was similar to CD133-CAR T cells.

\section{Galunisertib did not affect the proliferation of CD133-} CAR T cells, HER2-CAR T cells, and NT cells

To determine the regulatory effect of Galunisertib on $\mathrm{T}$ cell proliferation, CFSE-labeled T cells and irradiated target cells (or T cell activator) were co-cultured, and $0.1 \mu \mathrm{M}, 1 \mu \mathrm{M}$, or $10 \mu \mathrm{M}$
Galunisertib was added. The control group included DMSO and R10. U251 CD133-OE luc, MDA-MB-453 luc, or Transact was used to activate CD133-CAR T cells, HER2-CAR T cells, or NT cells, respectively. After four days, CFSE signals were analyzed by flow cytometry to analyze cell proliferation. Figure 6A shows that NT cells activated with Transact proliferated dramatically compared to unstimulated control; $0.1 \mu \mathrm{M}, 1 \mu \mathrm{M}$, or $10 \mu \mathrm{M}$ Galunisertib had no significant effect on the proliferation of NT cells, CD133-CAR T cells, and HER2-CAR T cells (Figure 6 B-D).

A

CD133-CAR T(E:T=2:1)
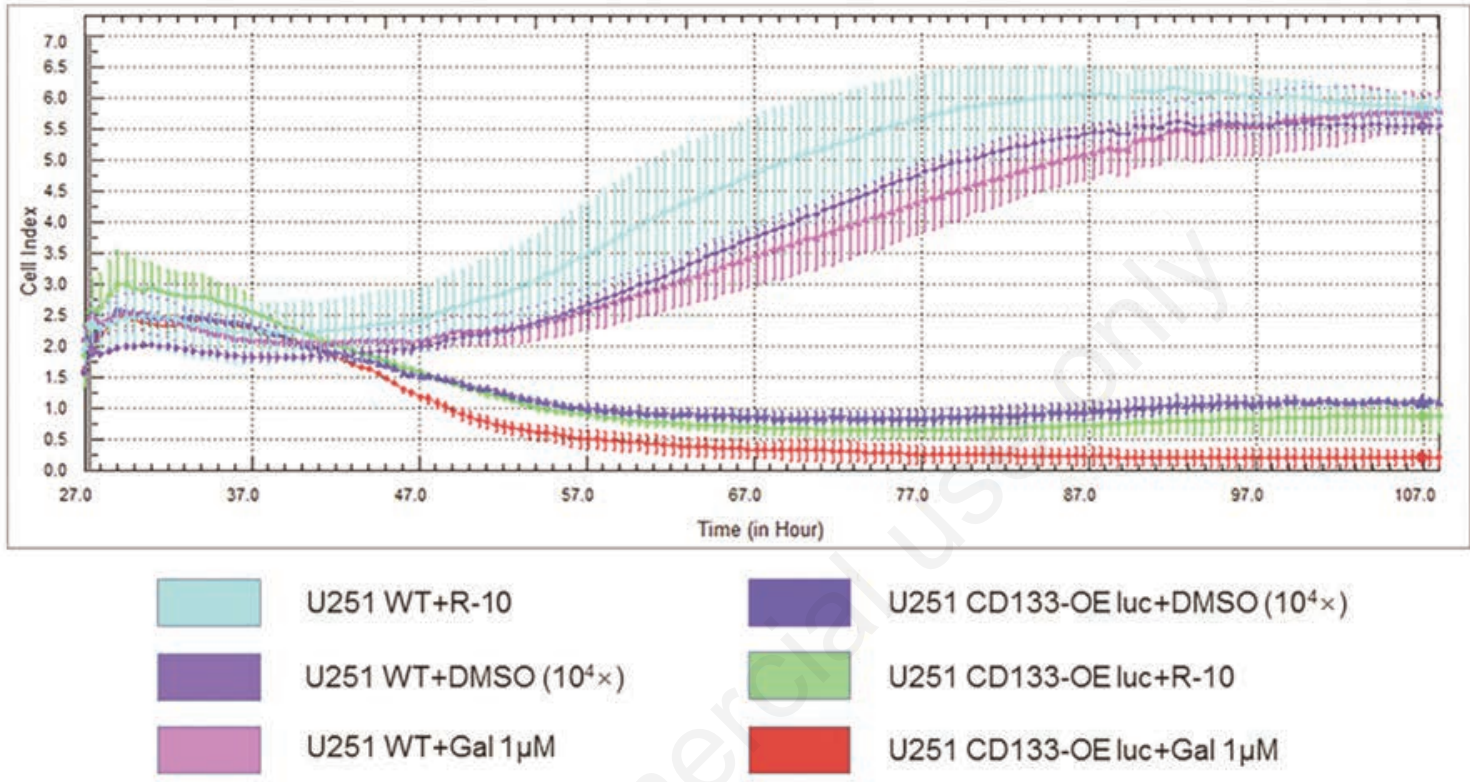

U251 CD133-OEluc+DMSO $\left(10^{4} \times\right)$

U251 CD133-OE luctR-10

U251 CD133-OE luc+Gal $1 \mu \mathrm{M}$

B

HER2- CAR T(E:T=1:1)

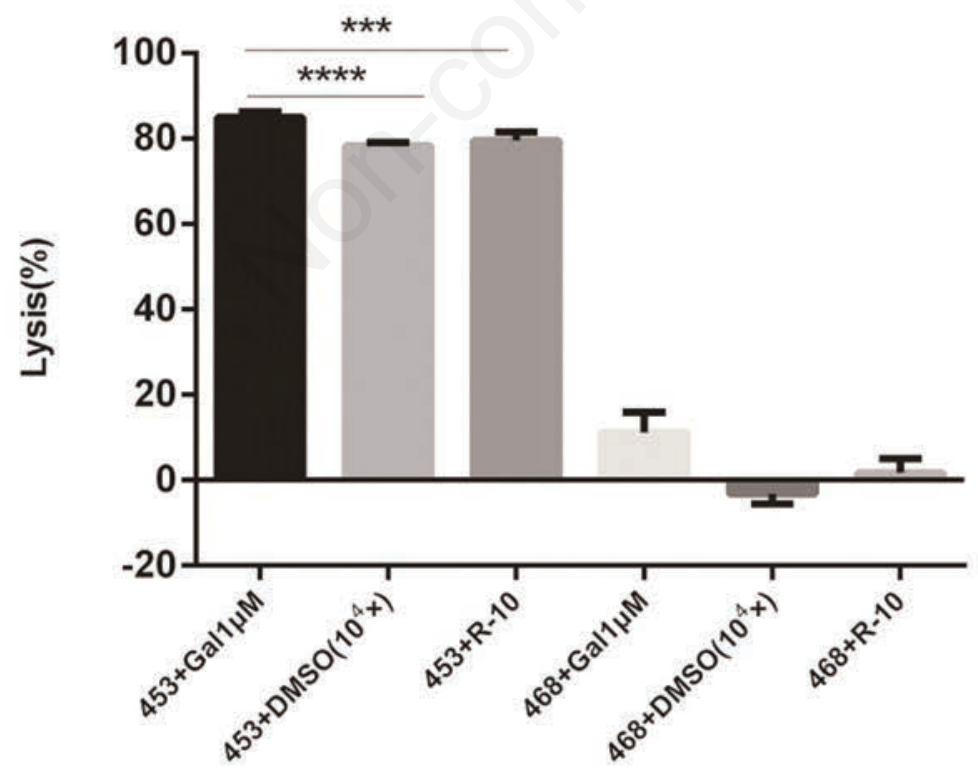

Figure 4. Galunisertib significantly enhanced the specific killing of tumor cells by CAR T cells. A) U251 CD133-OE luc or U251 WT cells were seeded into E-plate. One day later, CD133-CAR T cells and $1 \mu \mathrm{M}$ Galunisertib (Gal) were added, and DMSO or medium (R-10) was used as control. Cell viability (Cell Index) of tumor cells was monitored in real-time. B) HER2-CAR T cells were co-cultured with different target cells (MDA-MB-453 luc or MDA-MB-468 luc), and $1 \mu \mathrm{M}$ Galunisertib (Gal) was added simultaneously; $24 \mathrm{~h}$ later, the lysis rate was determined. 
Galunisertib regulating CAR $\mathrm{T}$ cell function may be achieved by blocking TGF- $\beta 1$-mediated signaling

To investigate whether Galunisertib's effects on the cytotoxicity and cytokine secretion of CD133-CAR T cells and HER2-CAR $\mathrm{T}$ cell were mediated by blocking TGF- $\beta 1$ signal, we first examined the level of TGF- $\beta 1$ in the cell supernatant of the co-culture system. CD133-CAR T cells or HER2-CAR T cells were co-cultured with U251 CD133-OE luc or MDA-MB-453 luc cells, respectively. NT cells were activated with Transact. The supernatant in the culture was collected $24 \mathrm{~h}$ later, and TGF- $\beta 1$ was measured by ELISA. TGF- $\beta 1$ secretion was clearly detected in all the three co-cultures (Figure 7).
A

CD133-CAR T
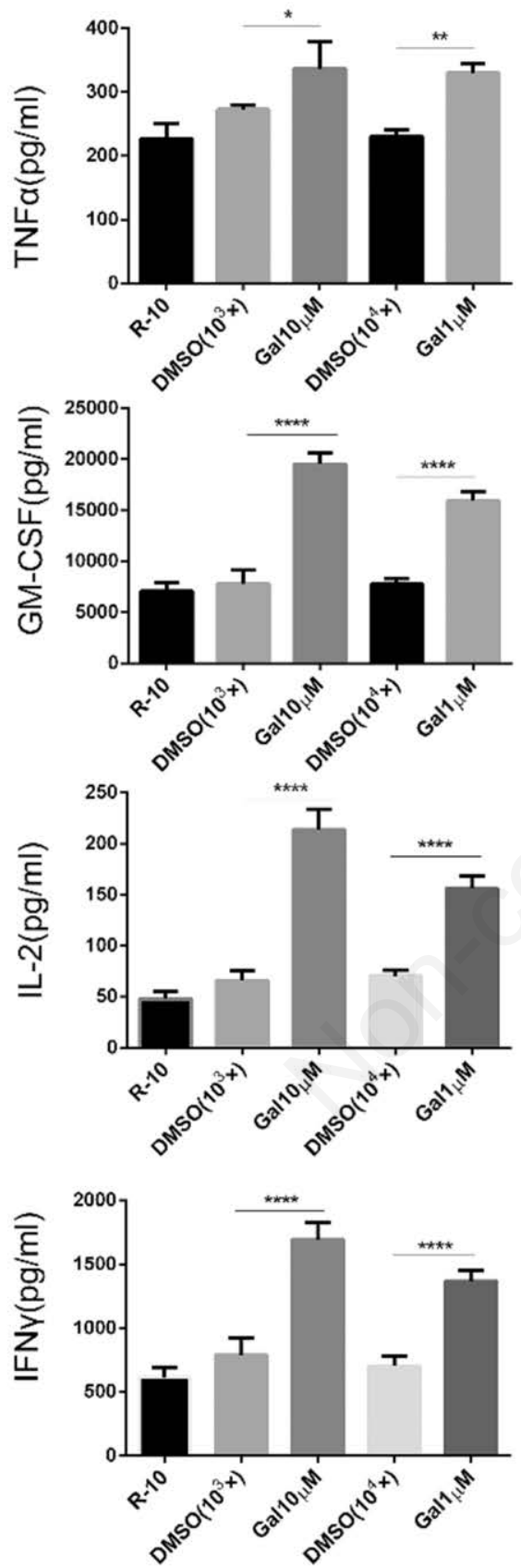

B

HER2-CAR T
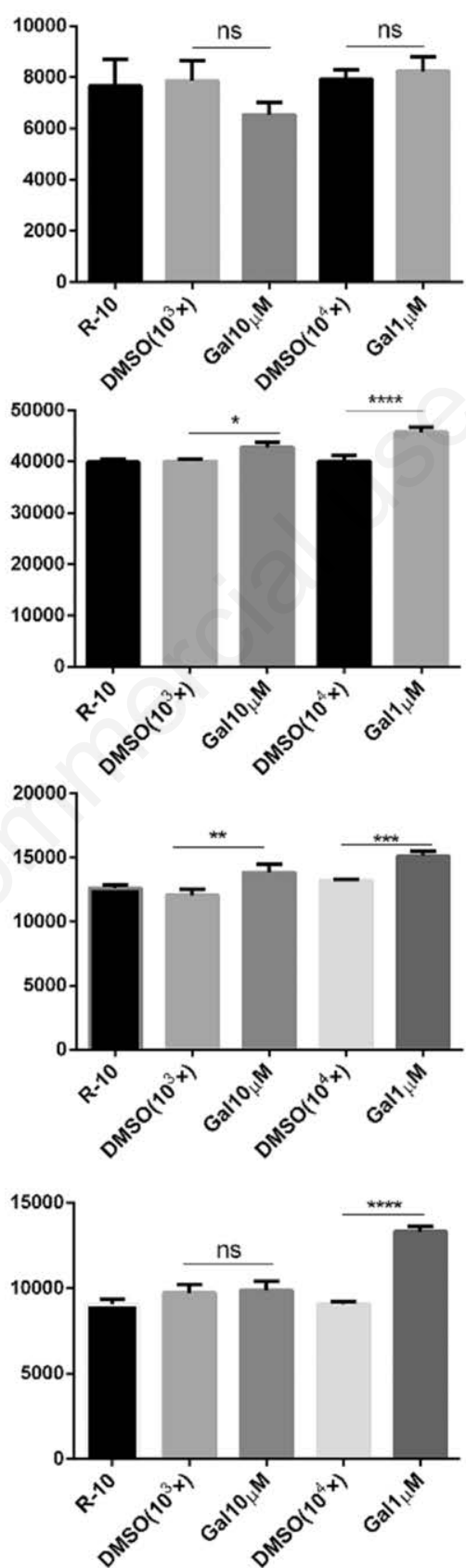

C
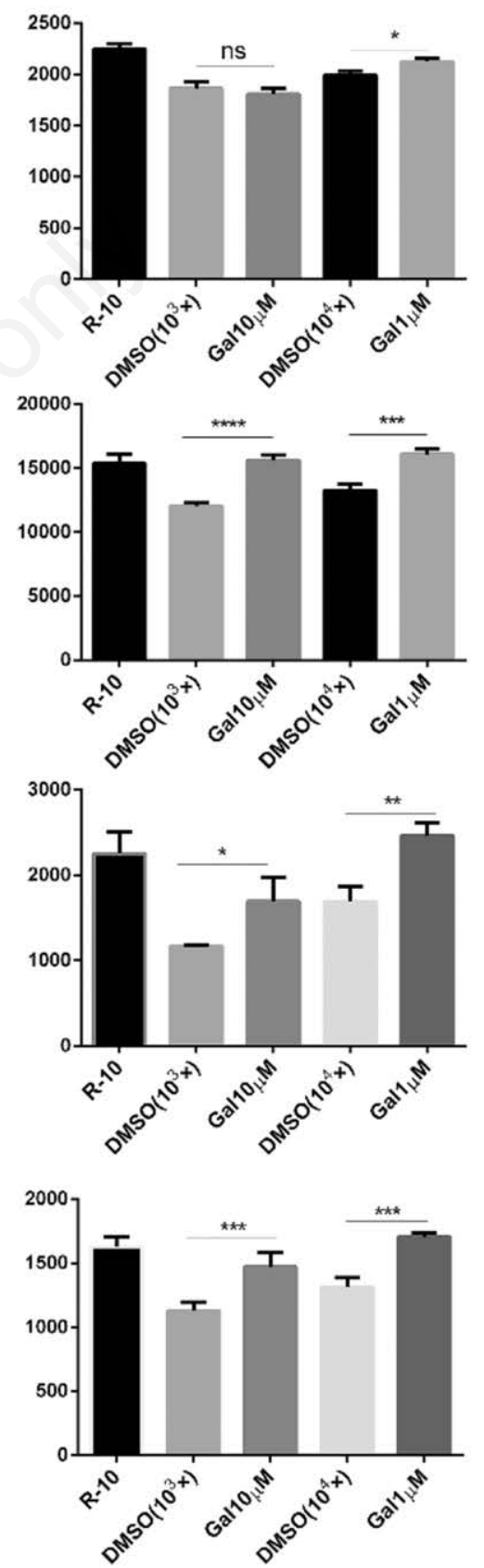

Figure 5. Galunisertib significantly increased cytokine secretion of T cells. Galunisertib (Gal) was added into (A) the co-culture of CD133-CAR T cells and U251 CD133-OE luc cells or (B) the co-culture of HER2-CAR T cells and MDA-MB-453 luc cells. C) NT cells were stimulated with Transact, while Galunisertib was added. After co-culture for $24 \mathrm{~h}$, the supernatants were collected and tested for the secretion of four cytokines (TNF $\alpha$, GM-CSF, IL-2, and IFN $\gamma$ ) using AlphaLISA. 
A
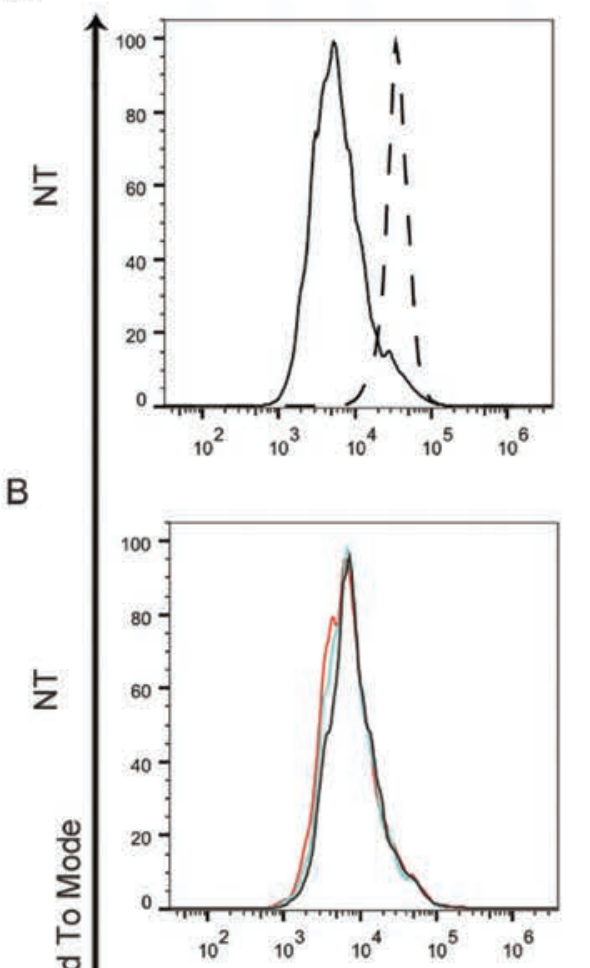

C

เ
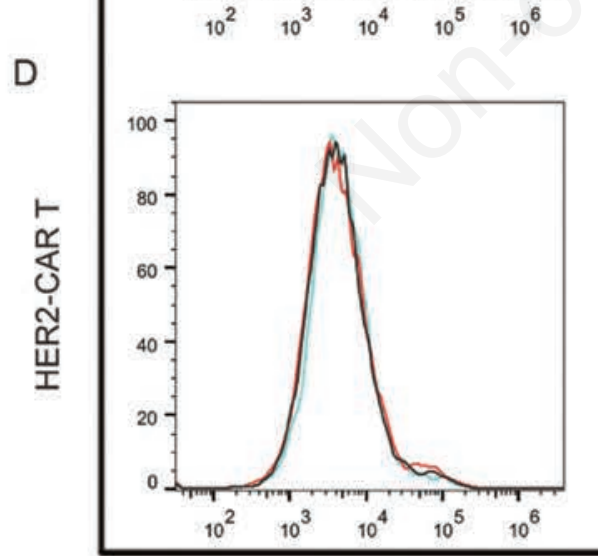

$\mathrm{DMSO}\left(10^{3} \times\right)$

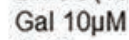

R-10
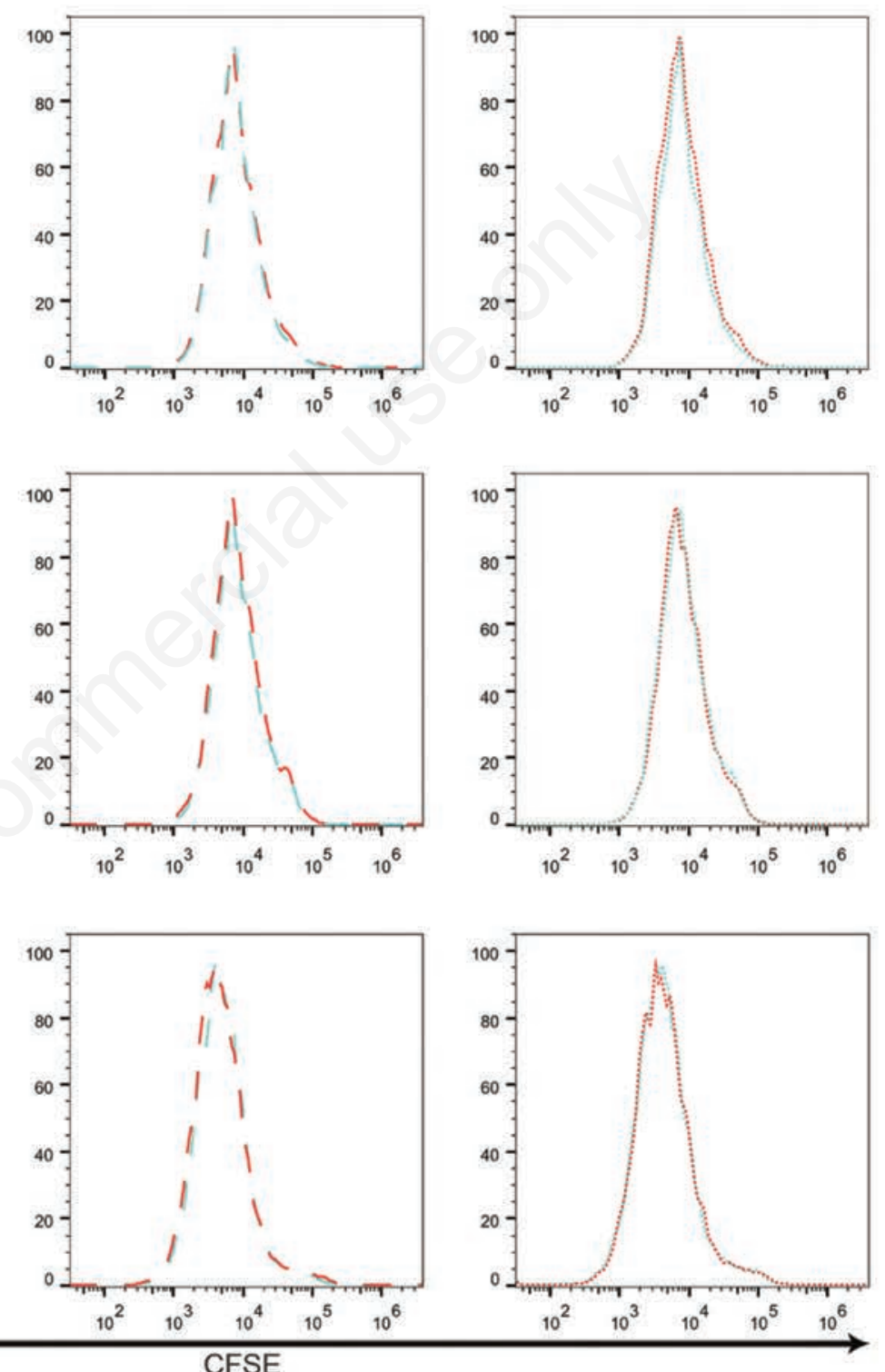

DMSO $\left(10^{4} \times\right)$

Gal $1 \mu \mathrm{M}$

$\operatorname{DMSO}\left(10^{5} \times\right)$

Gal $0.1 \mu \mathrm{M}$

Figure 6. Galunisertib did not affect T cell proliferation. (A) and (B) NT cells, (C) CD133-CAR T cells, and (D) HER2-CAR T cells were labeled with $0.5 \mu \mathrm{M}$ CFSE. (A) NT cells were cultured with (+Transact) or without Transact (control). B-D) Different T cells were activated with Transact, irradiated U251 CD133-OE luc and MDA-MB-453 luc cells, respectively. Meanwhile, Galunisertib was added, and DMSO or culture medium (R-10) was used as control. After four days, CFSE signals in T cells were detected by flow cytometry. 
In addition, Galunisertib did not significantly regulate the TGF- $\beta 1$ secretion by $\mathrm{T}$ cells.

Previous mechanism studies have shown that TGF- $\beta 1$-mediated signaling can cause phosphorylation of $\operatorname{Smad} 2 / 3$. To confirm the molecular mechanism of the action of Galunisertib, western blotting was used to detect phosphorylated Smad3 in T cells after treatment with $0.1 \mu \mathrm{M}, 1 \mu \mathrm{M}$, or $10 \mu \mathrm{M}$ Galunisertib for $24 \mathrm{~h}$. As shown in Figure $8,10 \mu \mathrm{M}$ Galunisertib could significantly reduce the amount of phosphorylated Smad3 protein in CD133-CAR T cells and HER2-CAR T cells, indicating that Galunisertib may promote $\mathrm{T}$ cell function by blocking TGF- $\beta 1$-mediated signals.

\section{Galunisertib has no effect on the expressions} of antigens on target cells and activation markers on CAR T cells

To investigate whether Galunisertib enhancing CAR T cell function was caused by increasing target antigen expression on tumor cells, we treated U251 CD133-OE luc and MDA-MB-453 luc with Galunisertib, DMSO or cell medium (D-10). And then we used flow cytometry to detect CD133 or HER2 on tumor cells. The results show that Galunisertib has no effect on antigen expression levels (Figure S1). To further study whether Galunisertib affect CAR T cell activation, CD133-CAR T cells or HER2-CAR T cells were activated with U251 CD133-OE luc or MDA-MB-453 luc cells, respectively. Meanwhile, Galunisertib was added, and DMSO or culture medium (R-10) was used as control. After two days, CD69 on T cells were detected by flow cytometry. As shown in Figure S2, Galunisertib has no effect on activation marker expression of CAR T cells.

\section{Discussion}

This study has shown that Galunisertib could enhance the cytotoxicity of CD133- or HER2-CAR T cells against U251 CD133OE luc or MDA-MB-453 luc cells in vitro. Moreover, Galunisertib promoted cytokine secretion by CD133-CAR T cells, HER2-CAR T cells, and NT cells. Galunisertib promoted TNF $\alpha$ secretion from CD133-CAR T cells and NT cells, but did not affect HER2-CAR $\mathrm{T}$ cells. The difference in the effects of Galunisertib on different CAR T cells may be related to molecular signals in CAR T cells or tumor cells that were used to activate CAR T cells. The activated NT cells secreted TGF- $\beta 1$, and the expression of TGF- $\beta 1$ was also detected in the co-culture of CD133-CAR T cells or HER2-CAR T cells with their target cells. Galunisertib enhanced T-cell killing of tumors may be achieved by blocking TGF- $\beta 1$-mediated signals.

The binding of TGF- $\beta$ ligand to TGF- $\beta$ receptor II (TGF- $\beta$ RII) initiates the TGF- $\beta$ signaling pathway. Subsequently, TGF- $\beta$ recep-
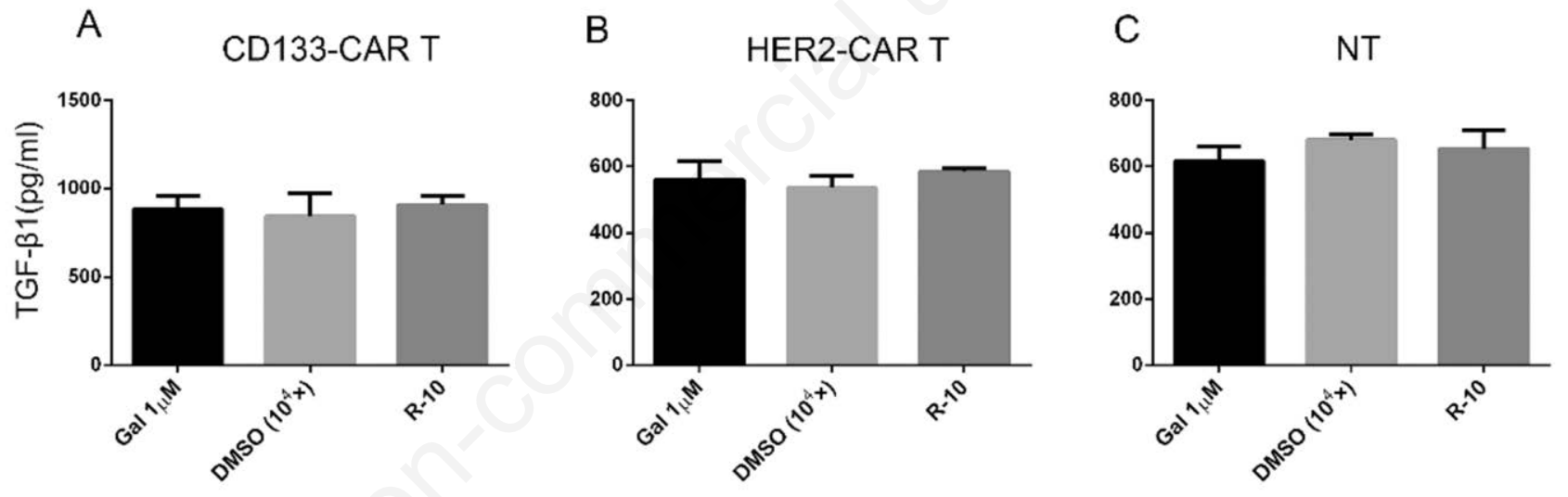

Figure 7. T cells secreted TGF- $\beta 1$ upon activation. (A) CD133-CAR cells and (B) HER2-CAR T cells were activated with U251 CD133OE luc and MDA-MB-453 luc cells, respectively. C) NT cells were stimulated with Transact. After co-culture for $24 \mathrm{~h}$, the supernatants were collected and the TGF- $\beta 1$ secretion was detected by ELISA.

Cell type CD133-CAR T

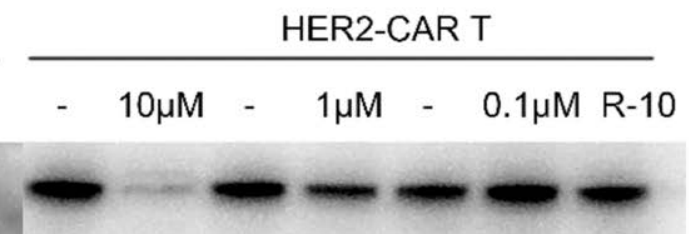

Smad3

$40 K D$

$35 \mathrm{KD}$
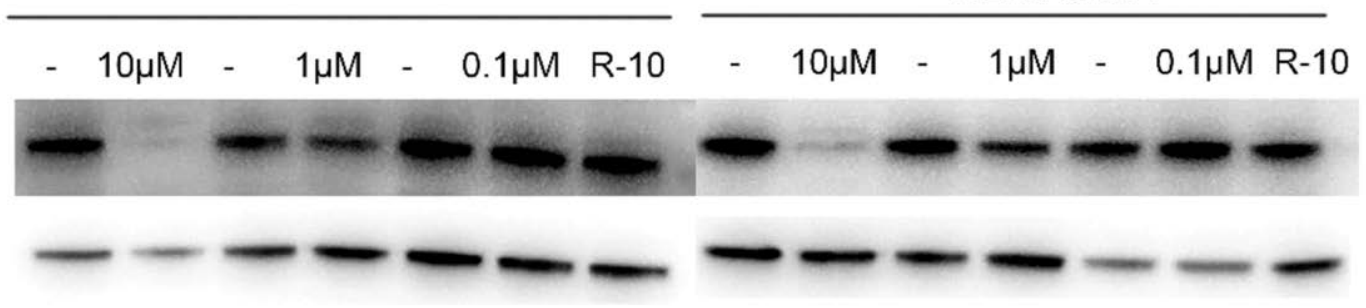

GAPDH

Figure 8. Galunisertib could block Smad3 phosphorylation in CAR T cells. CD133-CAR T and HER2-CAR T cells were treated with $0.1 \mu \mathrm{M}, 1 \mu \mathrm{M}$, or $10 \mu \mathrm{M}$ Galunisertib (Gal), with DMSO (-) and culture medium (R-10) as controls. After $24 \mathrm{~h}$, the amount of phosphorylated Smad 3 in CAR T cells was detected by western blotting. 
tor I (TGF- $\beta R I)$ forms a heterotrimeric complex with the ligand and TGF- $\beta$ RII. This complex phosphorylates serine/threonine kinase domains of TGF- $\beta$ RI, which subsequently activates the classical Smad2/3 signaling pathway and the non-classical mitogen-activated protein kinase (MAPK) pathway. ${ }^{15}$ Galunisertib (molecular formula: $\mathrm{C}_{22} \mathrm{H}_{19} \mathrm{~N}_{5} \mathrm{O}$; molecular weight: $369.42 \mathrm{Da}$ ) is an oral small-molecule inhibitor of TGF- $\beta$ RI. Galunisertib antagonizes TGF- $\beta$ signaling by blocking TGF- $\beta$ receptor-activated Smad phosphorylation. ${ }^{16}$ Studies have shown that Galunisertib could reduce the transmission of the TGF- $\beta$ downstream $\mathrm{Smad} 2 / 3$ signal in hepatocellular carcinoma, ${ }^{17}$ which was also found in ovarian cancer. ${ }^{18}$ In our study, Galunisertib significantly reduced Smad3 protein phosphorylation in CD133-CAR T cells, HER2-CAR T cells.

It was reported that Galunisertib inhibited the proliferation, migration, and invasion of high-grade serous ovarian cancer (HGSOC) cells ${ }^{18}$ in vitro. Galunisertib also inhibited the proliferation of microglia ${ }^{19}$ and breast cancer cell line $4 \mathrm{~T} 1 .{ }^{20}$ However, in this study, Galunisertib had no direct toxicity on glioma cell line $\mathrm{U} 251$ and breast cancer cell line MDA-MB-453 in vitro, which means that tumor cells we used are not sensitive to Galunisertib treatment.

TGF- $\beta$ inhibits the transcription of genes encoding multiple key proteins of the "cytotoxic program" (such as perforin and granzyme A, B, Fas ligand, and IFN $\gamma$ ), and thus the cytotoxicity of $\mathrm{CD}^{+} \mathrm{T}$ cells. ${ }^{21-23}$ In this work, it was further confirmed that the addition of Galunisertib, a TGF- $\beta$ receptor I inhibitor, to the culture system could enhance the ability of CAR T cells to kill tumors. TGF- $\beta 1$ inhibits IL- 2 production and TCR-mediated proliferation through the Smad3 pathway. ${ }^{24}$ In our study, Galunisertib could reduce the phosphorylation of Smad3 and promote the secretion of IL-2 by CD133-CAR T cells, HER2-CAR T cells, and NT cells. However, in the proliferation experiment, the addition of Galunisertib was not found to promote the proliferation of $\mathrm{T}$ cells. The reason for this may be that Galunisertib did not change the pathway that regulated $\mathrm{T}$ cell proliferation. Studies have shown that the mechanisms regulating killing and proliferation are different. For example, IL-2 could regulate the expression of perforin and granzyme genes in $\mathrm{CD} 8^{+} \mathrm{T}$ cells, which was not dependent on its effect on cell survival and proliferation. ${ }^{25}$ Research by Chen et al. also showed that $\mathrm{T}$ regulatory cells inhibited the cytotoxicity of $\mathrm{CD}^{+} \mathrm{T}$ cells through the TGF- $\beta$ receptor on the surface, but did not inhibit the proliferation of $\mathrm{CD}^{+} \mathrm{T}$ cells. ${ }^{26}$ The above points indirectly explain the reason that the addition of Galunisertib failed to promote $\mathrm{T}$ cell proliferation, but the killing function of $\mathrm{T}$ cells was improved.

The clinical trials using Galunisertib for treatment of myelodysplastic syndrome (MDS, phase II/III, NCT02008318), ${ }^{27}$ pancreatic cancer (phase II, NCT01373164), ${ }^{28}$ glioblastoma (phase II, NCT01582269), ${ }^{29}$ and hepatocellular carcinoma (stage II, NCT01246986) ${ }^{30}$ are ongoing. The initiation of those late-stage trials indicates that the clinical use of Galunisertib alone is relatively safe, which provides a safety study basis for the clinical treatment of solid tumors using Galunisertib combined with CAR T cells.

Resistance to Galunisertib often appeared in recruited patients, which limited its clinical application. ${ }^{31}$ In order to solve the problem, researchers have tried the combined use of Galunisertib and immune checkpoint inhibitors to treat tumors in clinical trials. For example, Galunisertib combined with Nivolumab is being used to treat hepatocellular carcinoma and non-small cell lung cancer (NSCLC) (NCT02423343), ${ }^{32}$ and with Durvalumab to treat pancreatic cancer (NCT02734160). ${ }^{33}$ In addition, given the immunosuppressive properties of TGF- $\beta$, Hou et al. designed CAR T cells specifically targeting TGF- $\beta$ to improve the anti-tumor immune responses. ${ }^{34}$ However, such CAR T cells that target TGF- $\beta$ could not specifically target tumor cells. We used Galunisertib in combination with CD133-CAR T cells, or HER2-CAR T cells, which may not only improve the suppression of immune microenvironment, but also specifically target tumor cells expressing CD133 or HER2, respectively.

In summary, Galunisertib promoted the functions of CD133CAR T and HER2-CAR T against solid tumor cells in vitro. This discovery provides a new strategy for small-molecule inhibitors combined with CAR T cells to treat tumor cells, and a basis for in vivo study of Galunisertib combined with CAR $\mathrm{T}$ cells in animal models. Based on our findings, clinical trials using the combination will be promising in the future.

\section{Acknowledgements}

We would like to thank the HTS Platform in SIAIS for the technical assistance. This work was supported by the National Key R\&D Program (2019YFA0111001) of China.

\section{References}

1. Ruella M, Kenderian SS. Next-generation chimeric antigen receptor T-cell therapy: Going off the shelf. BioDrugs 2017;31:473-81.

2. Kallam A, Vose JM. Recent advances in CAR-T cell therapy for non-Hodgkin lymphoma. Clin Lymphoma Myeloma Leuk 2019;19:751-7.

3. Shanghai GeneChem Co. Ltd. A study of GPC3 redirected autologous T cells for advanced HCC (GPC3-CART). Available from: https://clinicaltrials.gov/ct2/show/NCT0271 5362 ?term $=$ NCT02715362\&draw $=2 \&$ rank $=1$.

4. Zhi Yang RoBC, Southwest Hospital, China. A clinical research of CAR $\mathrm{T}$ cells targeting HER2 positive cancer. Available from: https://clinicaltrials.gov/ct2/show/NCT027 13984? term $=$ NCT02713984\&draw $=2 \&$ rank $=1$.

5. Haas AR, Tanyi JL, O'Hara MH, Gladney WL, Lacey SF, Torigian DA, et al. Phase I study of lentiviral-transduced chimeric antigen receptor-modified $\mathrm{T}$ cells recognizing mesothelin in advanced solid cancers. Mol Ther 2019; 27:1919-29.

6. Scarfo I, Maus MV. Current approaches to increase CAR T cell potency in solid tumors: targeting the tumor microenvironment. J Immunother Cancer 2017;5:28.

7. Pickup M, Novitskiy S, Moses HL. The roles of TGFbeta in the tumour microenvironment. Nat Rev Cancer 2013;13:788-99.

8. Lin JT, Martin SL, Xia L, Gorham JD. TGF-beta 1 uses distinct mechanisms to inhibit IFN-gamma expression in CD4+ T cells at priming and at recall: differential involvement of Stat 4 and T-bet. J Immunol 2005;174:5950-8.

9. Yingling JM, McMillen WT, Yan L, Huang H, Sawyer JS, Graff J, et al. Preclinical assessment of galunisertib (LY2157299 monohydrate), a first-in-class transforming growth factor-beta receptor type I inhibitor. Oncotarget 2018;9:6659-77.

10. Holmgaard RB, Schaer DA, Li Y, Castaneda SP, Murphy MY, $\mathrm{Xu} \mathrm{X}$, et al. Targeting the TGFbeta pathway with galunisertib, a TGFbetaRI small molecule inhibitor, promotes anti-tumor immunity leading to durable, complete responses, as monotherapy and in combination with checkpoint blockade. J Immunother Cancer 2018;6:47.

11. Herbertz S, Sawyer JS, Stauber AJ, Gueorguieva I, Driscoll $\mathrm{KE}$, Estrem ST, et al. Clinical development of galunisertib 
(LY2157299 monohydrate), a small molecule inhibitor of transforming growth factor-beta signaling pathway. Drug Des Dev Ther 2015;9:4479-99.

12. Hu B, Zou Y, Zhang L, Tang J, Niedermann G, Firat E, et al. Nucleofection with plasmid DNA for CRISPR/Cas9-mediated inactivation of programmed cell death protein 1 in CD133-specific CAR T cells. Hum Gene Ther 2019;30:446-58.

13. Zhu X, Prasad S, Gaedicke S, Hettich M, Firat E, Niedermann G. Patient-derived glioblastoma stem cells are killed by CD133-specific CAR T cells but induce the T cell aging marker CD57. Oncotarget 2015;6:171-84.

14. Morgan RA, Yang JC, Kitano M, Dudley ME, Laurencot CM, Rosenberg SA. Case report of a serious adverse event following the administration of $T$ cells transduced with a chimeric antigen receptor recognizing ERBB2. Mol Ther 2010;18:843-51.

15. Heldin $\mathrm{CH}$, Miyazono $\mathrm{K}$, ten Dijke P. TGF-beta signalling from cell membrane to nucleus through SMAD proteins. Nature 1997;390:465-71.

16. Yoon JH, Jung SM, Park SH, Kato M, Yamashita T, Lee IK, et al. Activin receptor-like kinase 5 inhibition suppresses mouse melanoma by ubiquitin degradation of Smad4, thereby derepressing eomesodermin in cytotoxic T lymphocytes. EMBO Mol Med 2013;5:1720-39.

17. Serova M, Tijeras-Raballand A, Dos Santos C, Albuquerque M, Paradis V, Neuzillet C, et al. Effects of TGF-beta signalling inhibition with galunisertib (LY2157299) in hepatocellular carcinoma models and in ex vivo whole tumor tissue samples from patients. Oncotarget 2015;6:21614-27.

18. Zhang Q, Hou X, Evans BJ, VanBlaricom JL, Weroha SJ, Cliby WA. LY2157299 monohydrate, a TGF-betaR1 inhibitor, suppresses tumor growth and ascites development in ovarian cancer. Cancers (Basel) 2018 7;10:260.

19. Bureta C, Setoguchi T, Saitoh Y, Tominaga H, Maeda S, Nagano S, et al. TGF-beta promotes the proliferation of microglia in vitro. Brain Sci 2019;10:20.

20. Liu FL, Mo EP, Yang L, Du J, Wang HS, Zhang H, et al. Autophagy is involved in TGF-beta1-induced protective mechanisms and formation of cancer-associated fibroblasts phenotype in tumor microenvironment. Oncotarget 2016;7:4122-41.

21. Trapani JA. The dual adverse effects of TGF-beta secretion on tumor progression. Cancer Cell 2005;8:349-50.

22. Thomas DA, Massague J. TGF-beta directly targets cytotoxic T cell functions during tumor evasion of immune surveillance. Cancer Cell 2005;8:369-80.
23. Russell JH, Ley TJ. Lymphocyte-mediated cytotoxicity. Annu Rev Immunol 2002;20:323-70.

24. McKarns SC, Schwartz RH, Kaminski NE. Smad3 is essential for TGF-beta 1 to suppress IL-2 production and TCR-induced proliferation, but not IL-2-induced proliferation. J Immunol 2004; 172:4275-84.

25. Janas ML, Groves P, Kienzle N, Kelso A. IL-2 regulates perforin and granzyme gene expression in CD8 $+\mathrm{T}$ cells independently of its effects on survival and proliferation. J Immunol 2005;175:8003-10.

26. Chen ML, Pittet MJ, Gorelik L, Flavell RA, Weissleder R, von Boehmer H, et al. Regulatory $\mathrm{T}$ cells suppress tumor-specific CD8 T cell cytotoxicity through TGF-beta signals in vivo. Proc Natl Acad Sci USA 2005;102:419-24.

27. Company ELa. A study of Galunisertib in participants with myelodysplastic syndromes. Available from: https://clinicaltrials.gov/ct2/show/NCT02008318?term $=$ NCT02008318\&draw $=2 \&$ rank $=1$.

28. Company ELa. A study in metastatic cancer and advanced or metastatic unresectable pancreatic cancer. Available from: https://clinicaltrials.gov/ct2/show/NCT01373164?term=NCT0 1373164\&draw $=2 \&$ rank $=1$.

29. Company ELa. A study in recurrent glioblastoma (GB). Available from: https:/clinicaltrials.gov/ct2/show/ NCT01582 269? term $=$ NCT01582269\&draw $=2 \&$ rank $=1$.

30. Company ELa. A study of LY2157299 in participants with hepatocellular carcinoma. Available from: https://clinicaltrials.gov/ct2/show/NCT01246986?term=NCT01246986\&draw $=2 \&$ rank $=1$.

31. Neuzillet C, Tijeras-Raballand A, Cohen R, Cros J, Faivre S, Raymond E, et al. Targeting the TGFbeta pathway for cancer therapy. PharmacoTher 2015;147:22-31.

32. Company ELa. A study of Galunisertib (LY2157299) in combination with Nivolumab in advanced refractory solid tumors and in recurrent or refractory NSCLC, or hepatocellular carcinoma. Available from: https://clinicaltrials.gov/ct2/show/ NCT02423343?term=NCT02423343\&draw $=1 \&$ rank $=1$.

33. Company ELa. A study of Galunisertib (LY2157299) and Durvalumab (MEDI4736) in participants with metastatic pancreatic cancer. Available from: https://clinicaltrials.gov/ct2/ show/NCT02734160?term=NCT02734160\&draw=2\&rank=1.

34. Hou AJ, Chang ZL, Lorenzini MH, Zah E, Chen YY. TGFbeta-responsive CAR-T cells promote anti-tumor immune function. Bioeng Transl Med 2018;3:75-86.

Received for publication: 15 March 2020. Accepted for publication: 11 April 2020.

This work is licensed under a Creative Commons Attribution-NonCommercial 4.0 International License (CC BY-NC 4.0).

CCopyright: the Author(s), 2020

Licensee PAGEPress, Italy

European Journal of Histochemistry 2020; 64(s2):3122

doi:10.4081/ejh.2020.3122 